\title{
Neutrons for scattering: What they are, where to get them, and how to deal with them
}

\author{
Thomas Ederth ${ }^{\star}$ \\ Division of Molecular Physics, IFM, Linköping University, 58183 Linköping, Sweden
}

\begin{abstract}
In neutron scattering studies of soft matter, a diverse array of methods and instruments are used, providing information on structure and dynamics on various length and energy scales. However, much of the infrastructure needed for neutron scattering is common for many instruments. After a brief historical retrospect of neutron scattering, this chapter introduces the basic infrastructure needed to conduct scattering experiments. This includes equipment that is used to produce, spectrally adjust and purify, and to deliver neutrons to the instruments where scattering experiments are conducted. The basics of the interaction of neutrons with matter is also introduced, as a preparation for the final sections on the different means at hand for neutron detection.
\end{abstract}

\section{Introduction}

\subsection{Neutrons as probes for the structure of matter}

Radiation from most of the electromagnetic spectrum is used to probe structure and excitations in matter, as befits the required length or energy scales. Neutron radiation have several advantages for such purposes: neutrons have (or, rather, as we will see, can be made to have) wavelengths comparable to interatomic and intermolecular separations (0.1-10 $\AA$ ), energies of the same magnitude as molecular excitations (1-100 meV), such as molecular vibrations or rotations, and lattice vibrations. Neutrons interact weakly with matter, which has several benefits: they penetrate deeply, so that buried interfaces and bulk samples can be studied, theoretical treatment can be simplified (for example by using the Born approximation and ignoring multiple scattering), and this permits elaborate sample environments and containers to be used. Neutron contrast varies in a non-systematic manner between nearby elements and isotopes, giving good contrast between elements of similar atomic weight, and enabling isotopic labelling. Neutrons also have a magnetic moment, and although studies of magnetism is rare in soft matter, several techniques for studying non-magnetic matter utilizes the magnetic properties of neutrons, e.g. spin-echo techniques and magnetic contrast layers for reflectometry. However, on the down side, we find that neutron sources have low brilliance, resulting in lengthy experiments, low signal-to-noise ratios, or requiring large samples; access is complicated and inconvenient in comparison to lab sources, and could involve several months' of waiting and perhaps lengthy travel. In addition, disregarding the cost of the large neutron facilities themselves, many experiments are still

^e-mail: ted@ifm.liu.se 
expensive due to the need for isotopes, large single crystals, large sample volumes, etc. As a consequence, exploitation of the unique properties and possibilities offered by neutrons is regularly done in parallel with extensive investigations with other, complementary, methods, such as scattering (static or dynamic light scattering, X-rays, electrons), scanning probe methods, nuclear magnetic resonance, and many other types of spectroscopy. Thus, neutron scattering methods are unlikely to solve many problems in soft matter science on their own. However, it is often the case, that neutrons can provide unique, and otherwise unavailable information, for solving a given problem. The tutorials in this volume provide tools to understand what neutron scattering is about, and how experiments are conducted, in order to help the reader identify those experiments where neutrons can make a considerable difference.

\subsection{Discovery of the neutron}

In retrospect, it is easy to overestimate and misrepresent the drama preceding important scientific discoveries, but the discovery of the neutron in 1932 clearly was one that was both anticipated and much needed. The existence of the neutron was predicted by Rutherford twelve years before the actual discovery, on the basis of experimental evidence. Theoretically, the description of atomic nuclei in terms of merely protons and electrons at the time, was associated with several difficulites and inconsistencies, which were resolved only when neutrons were introduced. The course of events behind this discovery is very briefly outlined in the following, and readers are referred to other sources for more detailed accounts [1]. In the early years of the 20th century, it was known that electrons were negatively charged, and that atoms were overall charge-neutral. In 1904, Thomson proposed what was to be known as the "plum pudding model" of the atom, where "...the atoms of the elements consist of a number of negatively electrified corpuscles enclosed in a sphere of uniform positive electrification..." [2]. The Geiger-Marsden experiment in 1909 [3], demonstrated the nuclear nature of atoms by observing the distribution of alpha particles deflected upon passing through a thin gold foil. Based on these observations, Rutherford presented an atomic model in 1911 [4], describing the atom as a small, positively charged, massive nucleus surrounded by a much larger cloud of negatively charged electrons. In 1920, Rutherford [5] suggested a more elaborate model in which the nucleus consisted of positive protons and neutrally-charged particles, e.g. a proton and an electron bound in some way ("nuclear electrons"). Rutherford called these particles neutrons. In 1932, Bothe and Becker [6] found that energetic alpha particles falling on certain light elements produced an unusually penetrating radiation, assumed to be gamma radiation since it was unaffected by electric charge. However, upon interaction with paraffin, Joliot-Curie and Joliot [7] found that this radiation lead to ejection of protons of very high energy. In the same year Chadwick $[8,9]$ performed similar experiments on beryllium, showing that the new radiation consisted of uncharged particles with about the same mass as the proton - these particles were indeed the neutrons that Rutherford had predicted about a decade earlier, and Chadwick was awarded the 1935 Nobel prize in physics for this discovery.

\subsection{The early years of neutron scattering}

Within four years of the discovery of the neutron, it had been recognized that neutrons have wave properties, that neutrons could be "thermalized" by collisions in paraffin, and that this radiation could be diffracted by crystalline solids, as demonstrated on powders by Halban and Preiswerk[10] and on single crystals by Mitchell and Powers [11]. The same year, the magnetic moment of the neutron was predicted, and Bloch [12] outlined many of the applications of magnetic neutron scattering that were to follow in the subsequent decades. The early experiments used "white" radiation radium-beryllium sources that were insufficient for quantitative scattering experiments, which had to await the advent 
of nuclear reactors in the late 1940s. In 1948 Wollan and Shull [13] published a number of powder diffraction patterns, obtained at the first full-scale nuclear reactor, the "Clinton pile" at Oak Ridge. A perhaps more significant milestone for the development of neutron scattering was the publication of scattering length data for about 60 elements and isotopes in 1951 [14]. Since nuclear scattering lengths cannot be calculated from theory, the experimental quantification of these interactions enabled a more systematic investigation of the opportunities offered by neutrons. In particular, neutrons were used early on to investigate systems where X-ray scattering (which was at that time well established) was not appropriate, for example hydrogen-containing materials, for distinguishing elements with similar X-ray form factors, or indeed using the magnetic properties of the neutron. Brockhouse and Stewart pioneered the field of inelastic neutron scattering by determining a phonon dispersion curve with a prototype triple-axis spectrometer in 1955 [15]. (Brockhouse and Shull had to wait until 1994 before they were awarded a Nobel Prize for their contributions to neutron scattering.) The development of methods pertinent to soft matter occurred much later, with SANS determinations of protein [16] and polymer structure [17], chain conformation in bulk polymers [18], and the first reflectometry experiments yet another decade later [19]. Early applications of scattering will be introduced further on in this volume, in subsequent chapters relating to specific methods. For a more detailed account of the development of neutron scattering, the reader is referred to the anthology by Bacon, showing the diversity of the field in its early years [20].

\section{Basic properties of neutrons}

The neutron is a fermion, and thus has spin $1 / 2$, and is electrically neutral. The rest mass $m_{n}$ is 1.6749 $\times 10^{-27} \mathrm{~kg}$, or $1.00866 \mathrm{u}$, which is 1.00138 times the proton rest mass. The neutron magnetic moment is $-1.042 \times 10^{-9} \mathrm{~B}$, or $-1.913 \mu_{N}$, where $\mu_{N}$ is the nuclear magneton (negative since it is directed opposite to the spin). Free neutrons are unstable, and decay into a proton, an electron and antineutrino with a half-life of approximately $10 \mathrm{~min}: \mathrm{n} \rightarrow \mathrm{p}+\mathrm{e}^{-}+\bar{v}_{e}$. This decay is insignificant for most scattering experiment, since, as we shall see, the time needed for a free neutron to travel from the source to the detector, is of the order of milliseconds.

The charge neutrality means that the interaction with matter is weak, since there is no Coulomb interaction with electrons or nuclei, and the interaction with matter is governed by nuclear and magnetic interactions, as will be explained later. The neutron is not an elementary particle (but a nucleon) since it is composed of three quarks, but the internal degrees of freedom of the neutron are not relevant when used as a probe for structure or excitations of soft matter, since the energies involved in the excitation of the internal structure are far too large. The standard model of particle physics predicts a small electrical dipole moment of the neutron, though this has not been experimentally verified.

Neutrons are quantum objects whose behaviour can be understood via either their wave nature or particle properties. An integral part of the wave-particle dualism is that the wave and particle natures can be manifested at different occasions in the same experiment, though not simultaneously. As a nucleon, we are perhaps more familiar with the particle description, but the neutron is also associated with a velocity-dependent de Broglie wavelength, given by $\lambda=h / p$, where $h$ is Planck's constant and $p=m_{n} v$ is the momentum.

\section{Neutron production}

Neutrons are strongly bound in atomic nuclei, the binding energy per nucleon varies between 5 and $10 \mathrm{MeV}$ for all but the lightest elements, and only nuclear reactions can split free neutrons from nuclei. The neutrons used in much of the early experimental work in the 1930s were obtained by 
bombardment of beryllium with $\alpha$-particles, and while there are many types of neutron generators in various sizes available [21], the neutron sources that are used for condensed matter investigations are large facilites producing neutrons via fission and spallation. The earliest fission reactors were constructed for military purposes in the late 1940 s, specifically for nuclear weapon production, but the excess neutron fluxes that became available were quickly taken advantage of for the development of neutron scattering instruments. Reactors dominated among research neutron sources up the early 1970s, when the development of accelerator-based, pulsed spallation sources started. One important driving force for this was the efficiency enhancements expected with pulsed sources, where fewer neutrons are used more efficiently. For high-power sources, heat removal is a major obstacle, and in spallation sources, less heat is produced per fast neutron. A considerable difference in efficiency is seen upon comparing the $58 \mathrm{MW}$ reactor at ILL with the $160 \mathrm{~kW}$ pulsed source at ISIS, where the latter runs at about $0.3 \%$ of the ILL power. The ESS spallation source under construction in Sweden is designed for $5 \mathrm{MW}$ (although it will initially be running at $2 \mathrm{MW}$ ).

Looking at the development of neutron facilities, there is currently a clear tendency in favour of spallation sources. There are certainly several technical and scientific reasons for this, but one should not ignore the political dimension. An increasing difficulty in opening new reactor sources is associated with mounting public opinion against nuclear reactors, and fission reactors are also burdened by nuclear proliferation concerns and more elaborate management of high-level radioactive waste. This has the effect that many of the reactor-based neutron sources that are being phased out as they reach the end of their technical lifetime, are not replaced, leading to an overall decline in the availability of neutrons. There are concerns that the current and planned pulsed sources will not have a total capacity that meets the needs from the scientific community, with ensuing decline in both competence and scientific activity in the field.

\subsection{Reactor sources}

Reactors for neutron research produce neutrons by fission of ${ }^{235} \mathrm{U}$. After the uptake of a slow neutron, the unstable ${ }^{236} \mathrm{U}$ atom splits into two fragmets (of variable mass), and in addition generating 23 neutrons, $\beta$ and $\gamma$ radiation and on average ca $180 \mathrm{MeV}$ kinetic energy. The emitted 'prompt' neutrons (emitted within $10^{-14} \mathrm{~s}$ ) can induce fission in other nuclei, but usually do this after having reduced their speed via collisions, to kinetic energies where uptake by $U$ is more probable. This is achieved by a moderating medium (see section 4), such as water. The reactor at ILL uses the cooling water as the primary moderator, but this is surrounded by a heavy water moderator, reflecting neutrons back into the core again. Many fission fragments also contribute to neutron production via 'delayed' neutrons emerging from their further radioactive decay; these neutrons together with the prompt neutrons sustain the chain reaction that maintains neutron production, if there is sufficient amount of available fissile material, and the delayed neutrons, emitted orders of magnitude later than the prompt neutrons, are essential for controlling the reactor.

Reactors steadily produce neutrons with a broad energy distribution, and are thus said to operate in continuous mode, delivering a steady stream of neutrons to the instruments. If neutron pulses are desired, they can be obtained via choppers (see section 6.2). The neutron spectrum produced by reactors have a Maxwellian energy distribution. The large flux of cold neutrons is suitable for large objects and slow dynamics, and reactor sources have the advantage that resolution can easily be tailored to specific experimental requirements.

Reactor sources are, as a rule, run as continuous sources with the chain reaction maintained at a steady state. The pulsed reactor IBR-2 in Dubna is an exception, and its main difference from other reactors consists in mechanical reactivity modulation by rotating neutron reflectors, ensuring that criticality is reached only when the reflectors coincide near the reactor core, generating power 
pulses with a power of $1850 \mathrm{MW}$ at a rate of 5 or $10 \mathrm{~Hz}$, with a pulse half-width of $340 \mu$ s for thermal neutrons.

\subsection{Spallation sources}

Spallation is a process where high-energy particles, usually protons at GeV energies, hit a target of neutron-rich material, such as heavy metals like tungsten, mercury, tantalum or uranium. The nuclei are highly excited by the collisions, and disintegrate under rapid evaporation of neutrons. These neutrons proceed to excite nearby nuclei, promptly emitting further neutrons, until the process decays. In spallation, the wavelength of the generated neutrons is shorter than the dimensions of the nuclei, resulting in internal excitation of nuclides (intra-nuclear cascade), which further increases the number of emitted neutrons. The emitted neutrons cover a wide energy spectrum with a peak around $2 \mathrm{MeV}$, but with a continuous distribution toward higher energies, rich in hot neutrons, with a small fraction emerging with nearly the incident proton energy, as a result of near-elastic collisions. With the exception of the latter contribution, the spectrum is not too different from a prompt fission spectrum, but in practice most neutrons will have collided several times before leaving the target, and the extractable spectrum is degraded. The spallation process is extremely short, and neutron emission decays within $10^{-15} \mathrm{~s}$, and is determined by the pulse length of the proton source. Pulse lengths of the order of $\mu \mathrm{s}$ result in short and large-amplitude neutron pulses with very good wavelength resolution for thermal neutrons, and short-pulse spallation facilities operate at frequencies in the range 10-60 Hz. To achieve high proton beam intensities, a linear accelerator is used to pump protons into a synchrotron, where a large number of proton pulses are injected, and accumulated, before being extracted to the target. For these short-pulse ${ }^{1}$ sources the induced thermo-mechanical stress in the target is considerable, and several advantages follows from extending pulse lengths to the ms range [22]: With long pulses, the total proton beam energy per pulse can be higher, resulting in an increased neutron flux, but at the expense of reduced resolution (introducing uncertainty in the flight time). This favours applications such as many small-angle scattering or reflectometry experiments in soft matter, which are not dependent on high wavelength resolution. An additional aspect is the considerable costs involved in these facilities. Long-pulse sources do not need a storage ring, and by using only a linear accelerator, a given proton beam power delivered to the target can be achieved at substantially lower cost by using only long pulses. At a lower repetition rate, these will also enable operation of large bandwidth instruments, considerably extending the range of accessible wavelengths, see the comments below on time structure. Major spallation sources in operation today are short-pulse facilities (ISIS, SNS, J-PARC). The planned second target station at SNS, and the ESS facility currently under construction near Lund in southern Sweden, are both long-pulse stations. ESS is designed for $1.3 \mathrm{GeV}$ protons and a $5 \mathrm{MW}$ average beam power, from $2 \mathrm{~ms}$ pulses at $16.6 \mathrm{~Hz}$, and using a rotating Tungsten target to handle the released thermal energy more effectively.

Thus, spallation produces neutrons of different energies, and since these have different velocities, they arrive at the detector at different times. Hence, if the arrival time of each neutron on the detector is recorded beside the scattering angle, the corresponding wavelength, and hence the scattering vector $q$ can be determined for each neutron. This is the time-of-flight mode, and the widespread use of this mode at spallation sources is the reason why instruments there are all end-of-tube instruments, which is usually not the case at reactor sources.

Due to their pulsed nature, spallation sources are naturally suited for time-of-flight instruments, and from the neutron energy distribution, particulary good for measurements at large energies. The

\footnotetext{
1 "Short" and "long" could be viewed in relation to the moderation time. Neutrons need of the order of $100 \mu$ s for thermal equilibration, and pulse lengths substantially shorter than this moderation time will result in similar moderated pulse shapes.
} 


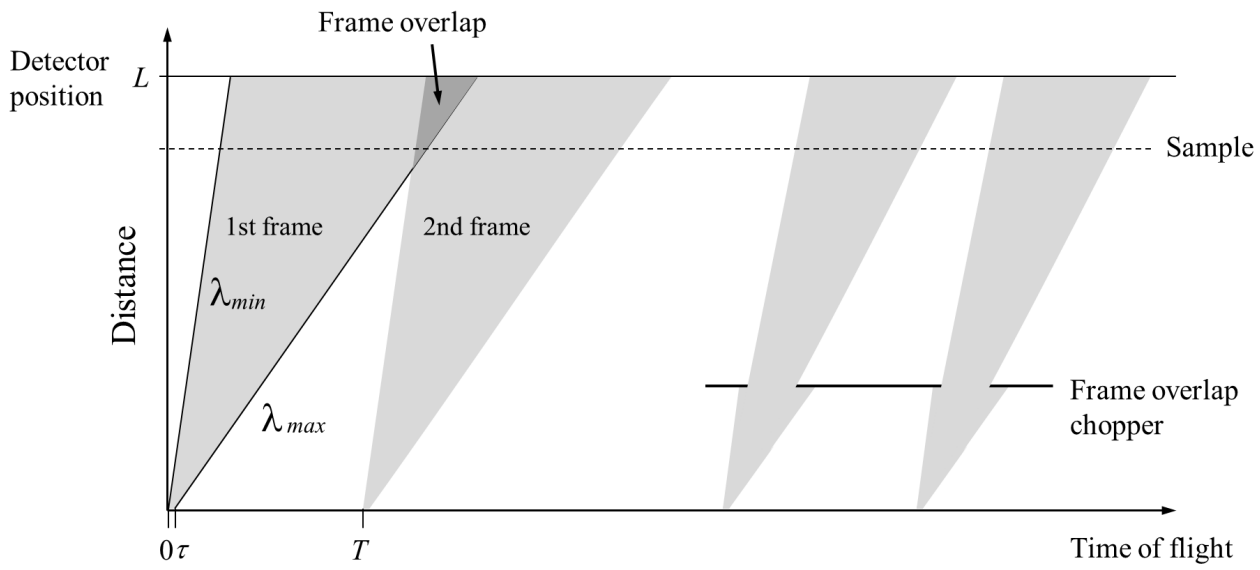

Figure 1. A time-of-flight diagram for a pulsed source generating a pulse of length $\tau$ every $T$ seconds. The diagram is shown without choppers (left) and with inserted pulse-defining choppers (right) whose purpose is to separate neutrons from subsequent pulses on the detector.

Swiss spallation source SINQ is unusual in that it is not a pulsed source; the neutron target is bombarded with protons from a cyclotron, delivering a quasi-continuous proton beam, making SINQ a continuous neutron source.

A very important property of pulsed sources is the time structure, that is, the duration and frequency of the pulses, from which time-of-flight diagrams can be constructed. Figure 1 illustrates how the source-detector distance $L$ and the pulse rate $(1 / T)$ will define the bandwidth of the instrument. The width of the pulse, $\tau$ can be ignored for short-pulse sources, but might noticeably affect the resolution on a long-pulse source. From the diagram it is clear that by increasing $L$, the resolution can be increased, but this must be accompanied by a lower pulse rate, to keep subsequent pulses separated on the detector, alternatively, overlap of two pulses ("frames") can be prevented with pulse-defining choppers, or with a high-pass filter, removing undesired slow neutrons, but at the same time reducing the bandwidth. Frame overlap must be avoided since the slow neutrons from the previous frame cannot be distinguished from the fast neutrons from the next frame. The leading and trailing edges of the frame are defined by the shortest $\left(\lambda_{\min }\right)$ and the longest $\left(\lambda_{\max }\right)$ wavelengths, and define the accessible $q$-range at a given scattering angle.

\subsection{Continuous versus time-of-flight operation}

Whether neutrons are produced continuously (as is usually the case in reactors) or in pulses (most spallation sources) has several consequences for the type and design of the scattering instrumentation used at the facilities. At continuous sources the beam is usually monochromatized for scattering experiments. This can be achieved by inserting a reflecting monochromator (see section 6.1) into the beam to deflect a specific wavelength to a diffractometer. Other wavelengths can then be extracted from the resulting beam downstream, or a velocity selector can be used at the guide end. The experiments are angle-dispersive, and the scattering vector $q$ is varied by varying the angles of the scattering experiment under the monochromatic beam, with data collected in $\theta / 2 \theta$-scans. At pulsed sources it would be very wasteful to use only neutrons of a particular wavelength in each pulse, and it makes 
more sense to use all neutrons, but to keep track of the energy (wavelength) of each neutron. In the latter case, the instruments are static, that is, kept at a fixed angle, and operated in time-of-flight (ToF) mode. This difference has several implications for the design and function of the instruments and the data collection systems, and also for sample environments. This does not visibly affect a SANS instrument much, but for reflectometry it often makes a difference whether the scattering plane is horizontal or vertical, or if angles are fixed or scanned during data collection; not all these options are compatible with, for example, experiments at air-liquid interfaces, to give an obvious example. The fundamentally very different operating principles of reactors and spallation sources have far-reaching implications on many aspects of scattering experiments, from generation, moderation and spectral distribution, to instrument layout and geometry, resolution and data treatment, and are not easily summarized (there is a more elaborate discussion on this topic in section 3.6 of ref. [23]). This pertains also to comparisons of the neutron flux from different types of sources. The time-averaged neutron flux is used to measure the neutron intensity of reactor sources, but how does this compare to the timeaveraged or the peak flux of a pulsed source? For similar integrated neutron fluxes, pulsed sources are overall more effective for conducting scattering experiments than reactor sources, and hence the time-averaged flux of a reactor source is usually compared to the peak flux of a pulsed source.

However, the differences between pulsed and continuous sources are also leveled out by development of instrumentation and measurement modes, thereby blurring the distinction between the two types of sources. Some examples of this are reactor instruments (such as the ILL D17 reflectometer) which are equipped with both monochromators and choppers (see section 6.2) to allow selection between monochromatic and time-of-flight operation on the same instrument. The continuous spallation source at the PSI uses many "reactor type" angle-dispersive scattering instruments, but have also chopper instruments for white-beam ToF mode.

\section{Moderators and neutron temperature}

The energy spectrum of neutrons generated in either a reactor or in a spallation source is inappropriate for most scattering experiments since the maxima in their energy distributions lie in the $\mathrm{MeV}$ range, which is far too high. A $1 \mathrm{MeV}$ neutron has a speed of $14000 \mathrm{~km} / \mathrm{s}$, and the corresponding de Broglie wavelength is $30 \mathrm{fm}$, which is clearly too short to be useful for structural characterization of molecular matter. Hence, the energy distribution needs to be shifted into a range that is more useful for scattering purposes, see Table 1. This is accomplished via moderation, a process where neutrons are allowed to thermally equilibrate with a volume of material to reduce the average kinetic energy via inelastic collisions. This is most efficiently done with a light material with a large collision cross section but low absorption, such as light or heavy water, graphite or beryllium. Hydrogenous materials are common, since $\mathrm{H}$ has a very large scattering cross-section ( $82 \mathrm{barn}$; cross-sections are measured in the unit barn (b), where $1 \mathrm{~b}=10^{-28} \mathrm{~m}^{2}$ ), and a mass similar to the neutron mass, resulting in efficient inelastic dissipation of the kinetic energy. At thermal equilibrium, the neutron velocities are Maxwell-Boltzmann distributed, and translated into wavelength, the neutron beam flux $\phi(\lambda)$ (neutrons per second per unit area), in the wavelength interval $[\lambda, \lambda+d \lambda]$, is then

$$
\phi(\lambda) \propto \frac{1}{\lambda^{5}} e^{-h^{2} / 2 m_{n} k T \lambda^{2}}
$$

This distribution is shown for three different temperatures in Figure 2, and the peak in this distribution occurs at

$$
\lambda=\frac{h}{\sqrt{5 m_{n} k_{B} T}}
$$


Table 1. Neutron temperatures, the corresponding wavelengths and energies, and examples of moderator materials to achieve these temperatures.

\begin{tabular}{lccc} 
& Wavelength $(\AA)$ & Energy $(\mathrm{meV})$ & Example of moderator \\
\hline Fast neutrons & $<0.5$ & & \\
Hot neutrons & $0.5-1$ & $80-300$ & Graphite at $2000 \mathrm{~K}$ \\
Thermal neutrons & $1-5$ & $3-80$ & $\mathrm{D}_{2} \mathrm{O}$ at $300 \mathrm{~K}$ \\
Cold neutrons & $3-30$ & $0.1-10$ & Liquid $\mathrm{H}_{2}$ at $25 \mathrm{~K}$ \\
Ultracold neutrons & $>500$ & & \\
\hline
\end{tabular}

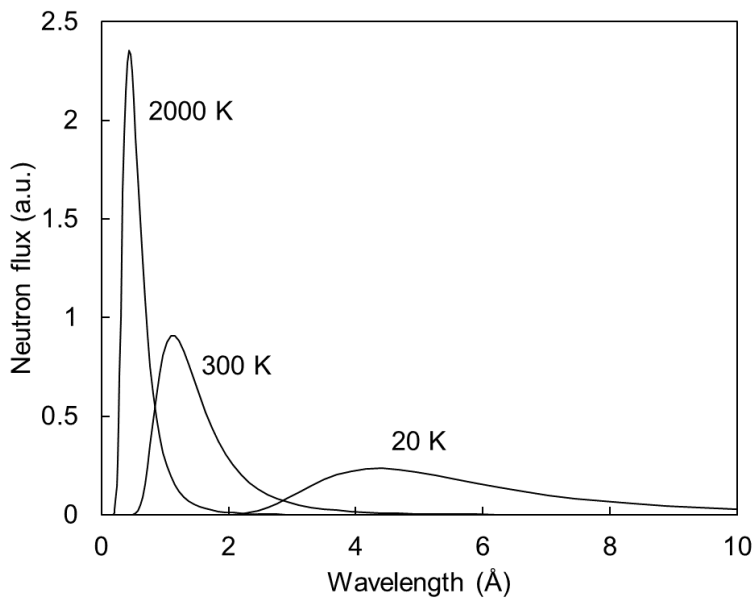

Figure 2. The Maxwellian wavelength distribution of neutrons in thermal equilibrium, at three different temperatures. The three distributions have the same area. At $300 \mathrm{~K}$, the mean neutron wavelength is $1.8 \AA$, and the mean energy $26 \mathrm{meV}$. For comparison, we note that photons with $\lambda=$ $1.8 \AA$ have an energy of $7 \mathrm{keV}$, and the temperature is $8 \times 10^{5} \mathrm{~K}$.

In reality, the energy dependence of the collision cross-section of most materials gives lower-energy neutrons a larger effective cross-section for a given nuclide than fast neutrons, which slightly modifies the energy distribution so that hotter neutrons are more abundant than predicted.

Depending on the incident neutron energy and the moderator material, 10-100 collisions are needed for equilibration, after which the neutrons diffuse isotropically in the moderator. The moderator has one or several exits, allowing the neutrons to enter as a beam into a neutron guide. The isotropic diffusion in the moderator allows the neutron guides to be aligned so that they are not directly facing the primary source, which reduces gamma radiation and uncollided high-energy neutron background in the beam.

Moderators are an intrinsic part of the design of a reactor, since fission neutrons must be moderated to be efficiently captured by ${ }^{235} \mathrm{U}$, often using heavy water, light water, or graphite, but there is no corresponding such function of the moderator for spallation sources. This means that there is more freedom in designing moderators to suit the needs of the instruments at a spallation source. On the other hand, spallation source moderators are preferably kept small to keep the pulse width narrow, resulting in under-moderation and an appreciable fraction of high-energy (epithermal neutrons). The smaller size also results in less efficient coupling to the neutron guides, while there are no such size constraints at reactor sources. As an example, the moderators are about 0.51 at the ISIS spallation source Target Station 1.

Thermal neutrons, with wavelengths in the range 1-5 $\AA$, which is of the order of interatomic and molecular distances, have energies around $30-700 \mathrm{~cm}^{-1}$, or about $3-80 \mathrm{meV}$, where many molecular and lattice excitation energies are found. However, for some experiments, especially reflectometry, 


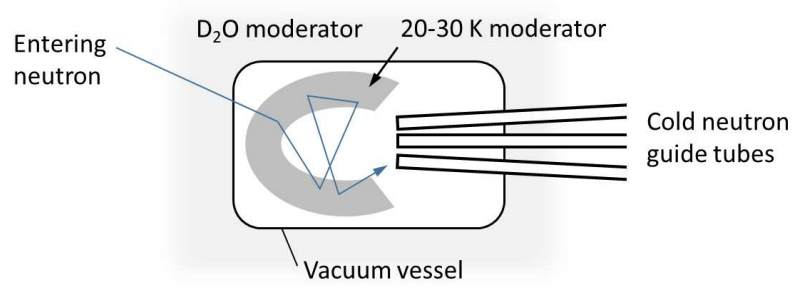

Figure 3. Schematic view of a cold source moderator. Neutrons enter from the surrounding $300 \mathrm{~K}$ moderator, typically containing $\mathrm{D}_{2} \mathrm{O}$. Neutrons with high energy have a greater probability (lower cross section) to pass through the cold moderator material, for example liquid hydrogen, into the cavity. Via repeated collisions the kinetic energy is reduced, and as the energy decreases, the cold moderator will be increasingly acting as a reflector, retaining neutrons in the cavity, until they enter one of the guide tubes.

small-angle scattering and biological applications, it is sometimes desirable to have neutrons of longer wavelengths, and these are produced in a cold source. This is commonly a hydrogen-containing material, such as hydrogen or methane kept at temperatures near 20-30 K, often surrounded by a Be reflector to bring diverted neutrons into the moderator, see Figure 3. On the other hand, neutrons with shorter wavelengths are obtained from a hot source. At the ILL this is a graphite block in the heavy water moderator which is heated to ca $2000 \mathrm{~K}$ by the collisions of hot neutrons with the graphite.

In pulsed sources, there is a constraint in that the pulse length needs to be kept short, and hence there is an upper limit to the time neutrons can be allowed to diffuse in the moderator before they enter the neutron guides. This is managed via decoupling the moderator with a neutron-absorbing material inserted between the moderator and the reflector material. Cadmium absorbs neutrons strongly, but as with most materials the absorption cross-section is wavelength-dependent, and it is almost transparent for energies above $0.3 \mathrm{eV}$. This means that fast neutrons from the spallation pulse can enter the moderator, but neutrons that have lost too much energy (spent too long time) in the reflector or in the moderator, are removed. The width of the moderator pulse can also be controlled via poisoning, where a small amount of neutron-absorbing material, such as gadolinium, is added to the moderator. This has a similar effect as decoupling; it narrows the pulse at low energies where the absorption cross-section is largest, and effectively clears the moderator of neutrons that have spent too long time in the moderator.

\subsection{Neutron guides}

Using guide tubes [24] to channel neutrons away from the neutron source allows a larger number of instruments to use the same source, since floor space in the immediate vicinity of the source does not limit the number of instruments, but allows instruments to be physically located further away, in guide halls. A location further from the neutron source reduces the radiation background, and placing instruments in a guide hall separated from a reactor source also means that users can access instrument under less strict radiation protection and safety regulations, which benefits both the users and the safety of the neutron facility.

Guide tubes are typically made of glass with neutron-reflective coatings lining the inside walls, with rectangular cross sections (width $1-5 \mathrm{~cm}$, height 5-20 cm). Neutrons travelling near-parallel to the guide walls are kept within the tube by total reflection from the walls. Most solids have neutron refractive indices which are lower than 1 for cold and thermal neutrons, and thus have a critical angle, $\theta_{c}$, below which neutrons are totally externally reflected (see section 7.2), and hence can be kept inside 
the guide tube with very low losses. The inside is coated with $\mathrm{Ni}$ or with $\mathrm{Ni}$-Ti heterostructures with hundreds of layers, or with supermirrors, [25] multilayer mirrors with gradually varying thicknesses of the layers, resulting in several times higher critical angles than plain Ni coatings (there are also polarized mirrors, using e.g. $\mathrm{Co} / \mathrm{Ti}$ or $\mathrm{Si} / \mathrm{Fe}$, and alternating ${ }^{58} \mathrm{Ni}$ and ${ }^{62} \mathrm{Ni}$ isotope layers). A multilayer coating introduces a Bragg reflection at a certain angle, and the gradient in the lattice spacing of supermirrors effectively produces a range of effective Bragg angles, and therefore a reflectivity which extends to several times the critical angle values expected for other coatings. This allows for curved guide tubes, and guides are usually given a slight curvature in the horizontal plane, because this has some benefits. A small curvature eliminates gamma radiation from the neutron source, since gamma radiation travels straight through the walls of the guides (and into surrounding shielding). Further, since neutron reflectivity is strongly wavelength dependent, the curvature can also be used to eliminate fast neutrons, and the radius can be used to tune the lower cutoff wavelength. This also makes guide tubes more useful the longer the wavelength, and thus much better for use with cold neutrons than for hot neutrons. Where there is not room enough for longer curved guide tubes, multichannel guides with several parallel supermirror plates inserted into the guide are used. These "beam benders" have a geometry like a curved Söller collimator (see next section). Recent developments in guide tubes include elliptic focusing guides, [26] which considerably improve flux at small sample areas and additionally reduce background scattering, since they allow tuning of the beam footprint to fit the sample size and hence minimize illumination of areas outside the sample. However, this will come at the cost of reduced resolution, since beam divergence at the sample increases.

The distance that neutrons can be transmitted in guide tubes is limited by gravity and reflection losses at the walls. For reactors and other continuous sources, there is in principle no limit to the length of a guide tube, except that gravity might have to be taken into account. For pulsed sources, the time structure of the source (the desired bandwidth) sets limits to the distance between the source and the instrument, to avoid mixing of neutrons from subsequent pulses. Guide lengths rarely exceed $100 \mathrm{~m}$, but several instruments at the ESS facility will be placed over $150 \mathrm{~m}$ from the spallation target. Neutron guides (and beam paths in general) are evacuated, to reduce losses due to scattering against air molecules, which reduces the flux of a neutron beam by approximately $5 \%$ per $\mathrm{m}$ at normal air pressure - and considerably more if the air is humid, due to the large hydrogen cross section.

\section{Collimation}

Collimators remove neutrons from the beam which do not travel in the desired direction, that is, they reduce beam divergence and increase the angular resolution $(\Delta \theta / \theta)$. This is achieved by placing slits or apertures (pinholes) in the beam path that absorb neutrons with too large angular deviation from the desired path, either in the primary beam, or before the detector. In principle, two pinholes would be sufficient, but in practice often more than two are used, to eliminate stray neutrons. Long collimation systems or small apertures obviously give the best collimation, but at the expense of reduced beam intensity, and vice versa. Again, this is a tradeoff between resolution and intensity that must be done for each experiment.

A Söller collimator is a system of parallel plates made of a neutron-absorbing material. Neutrons that hit the plates are absorbed, and the separation and the length of the plates determine the acceptance angle, see Figure 4. This results in collimation in one plane, but with slits or apertures, rectangular or circular collimation is obtained. Two slits before the sample, each limiting the extent of the beam both in the vertical and horizontal directions, one immediately before the sample, the other one or a few meters upstream, ensure that only the sample is illuminated, and that this illumination is a near-parallel beam. Beside maintaining the angular resolution, this also reduces scattering from surrounding equipment and sample holders. Similarly, slits between the sample and detector reduce 


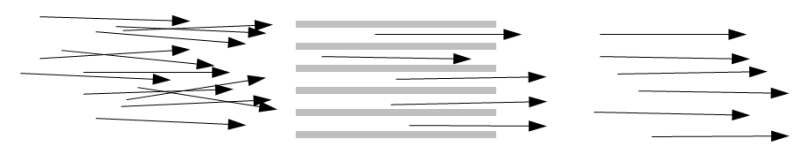

Figure 4. A Söller collimator is a stack of absorbing parallel plates, where the length $L$ and separation between the plates $d$ define the divergence $\alpha$ of the passing netrons, via $\alpha=d / L$.

background noise on the detector by minimizing the detection of neutrons scattered from sample holders. Slits are usually motorized since their openings are regularly varied with the incidence angles, and adjusted for sample size.

A radial collimator has absorbing plates arranged in a fan-shaped structure, these are used to reduce background on large-area detectors, and ensure that only neutrons from those parts of the sample which are both illuminated by the beam and which have a free line-of-sight through the collimator are counted.

\section{Monochromators}

The majority of the instruments at reactor sources use a fixed wavelength in angle-dispersive configurations for scattering experiments, and use monochromators for defining the wavelength or to analyze the energy of inelastically scattered neutrons. A conceptually simple way of preparing a monochromatic beam is to use the Bragg reflection from a single crystal of some suitable material, inserted into the guide. The Bragg angle $\theta_{B}$ is given by

$$
n \lambda=2 d \sin \theta_{B}, \quad n=1,2,3, \ldots
$$

where $n$ is the order of the reflection and $d$ the lattice spacing. However, the practical implementation of this principle is somewhat involved. The large size of the neutron guides means that the crystals need to be very large to cover the whole beam cross section. For all experiments there will also be a balance between the flux and the desired wavelength resolution, $\Delta \lambda / \lambda$. Permitting a lower resolution (larger $\Delta \lambda / \lambda$ ) means that the flux can be increased. Depending on the experiment, the required $\Delta \lambda / \lambda$ can vary from $10^{-4}$ to $10^{-1}$, which means that monochromators will have very different designs in order to fulfil these requirements. For many experiments in soft matter science, the resolution requirements are relatively moderate, and for small-angle scattering $\Delta \lambda / \lambda \approx 10 \%$ is not uncommon, and sometimes even $20 \%$ can be accepted, with associated benefits in higher available flux.

\subsection{Monochromator crystals}

Commonly used monochromator crystal materials are silicon, germanium, copper, pyrolytic graphite, or beryllium. A single crystal could be expected to yield a monochromatic beam with $\Delta \lambda / \lambda \approx 1 \%$. As mentioned, flux and resolution are important design parameters, and where lower resolution can be accepted, there are several ways of modifying crystals to increase the flux. A single crystal gives high resolution, but low flux. A simple way of increasing the flux is to increase the thickness of the crystal, or to place crystals after each other in the beam, though this compromises the angular resolution. If a single crystal is mechanically strained under heat, dislocations form in the crystal, and the local alignment of the lattice planes is slightly distorted. Introducing such mosaicity results in a spread of Bragg angles over the crystal volume, with deviations of the order of 30 arc minutes, and widens the 


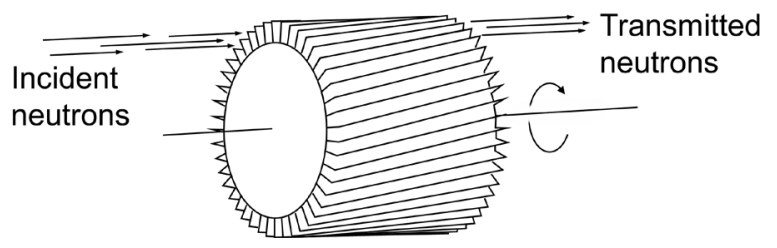

Figure 5. A velocity selector. The rotation speed and the helical pitch of the lamellae along the beam direction allows neutrons within a narrow velocity distribution to pass the selector.

reflected wavelength spread to approximately $5 \%$, but if this is tolerated it also increases the flux considerably. Alternatively, several crystals can be mounted at slightly varying angles, reducing the need for a large single crystal and allowing instead focussing of the beam. A single crystal that is slightly bent will also have a focussing effect, and at the same time some effective mosaicity since the actual Bragg angle will vary over the curvature.

\subsection{Choppers}

A chopper is a rotating disk made of neutron-absorbing material with at least one aperture, and is used as a shutter which allows beam transmission at certain time intervals. At spallation sources choppers are used to exclude certain wavelengths, and the rotation is synchronized with the pulsed beam (pulsedefining, or frame overlap choppers, see Figure 1). Neutrons of different energies arrive at the chopper at different times, and only those neutrons that arrive when the aperture is aligned with the beam pass through the aperture. In practice, the chopper is often assembled by two disks whose relative angles can be adjusted to allow the size of the aperture (the "angular window") to be adjusted. Two (or more) synchronized choppers separated along the rotation axis have a similar function as a velocity selector, but with greater flexibility since adjustment of both rotation speed and the relative angle of the two disks can be used to control the transmitted bandwidth. Choppers are also used to create pulses for time-of-flight experiments at reactor sources, though this is a wasteful mode of operation, in that only a small fraction of the white-beam neutrons are used.

\subsection{Velocity selectors}

A velocity selector can be thought of as a twisted and rotating Söller collimator, see Figure 5. Parallel plates arranged in a helical shape on the surface of a cylinder, with neutron-absorbing walls, permit neutrons to pass between the slits if they have a speed that is determined by the helical pitch and rotation speed of the cylinder. Velocity selectors offer lower wavelength resolution than Bragg reflectors, typically $10 \%$ (with ensuing increase in flux), and are most frequently used at end of neutron guide tubes at reactor sources, for e.g. SANS instruments.

\subsection{Filters}

Filters remove neutrons of unwanted energies from a beam either by deflecting them out of the beam, or by absorbing the neutrons. A pertinent example is higher order Bragg diffractions from a beam monochromator crystal. According to Bragg's law (Equation 1), a diffracted beam will also contain wavelengths of higher order ( $n>1$, giving $\lambda / 2, \lambda / 3$ etc.), and these need to be filtered away to obtain a monochromatic beam. Filters which diffract the unwanted wavelengths out of the beam direction are often graphite of liquid nitrogen-cooled beryllium, and should be selected so that $\lambda>2 d_{f}$, where $d_{f}$ is the lattice spacing of the filter crystal, so that the desired wavelength is not itself Bragg reflected. Suitable filters should have high coherent cross sections, but low incoherent and absorption cross 
sections. Filters are also cooled to minimize scattering of the transmitted beam by lattice vibrations (phonons) in the crystals.

Filters relying on (resonant) absorption are usually strongly absorbing in certain spectral regions. While Cd is widely used for shielding, and is very effective for thermal neutrons, but has limited absorption for fast neutrons. Certain isotopes with strong nuclear resonances are effective bandstop filters, and are used as thin foils, but most lie in regions of the energy spectrum that are of less interest to work in soft matter.

\section{Interactions of neutrons with matter}

In the absence of charge and associated Coulomb interactions, neutrons interact weakly with atoms, and do so via either of two mechanisms: magnetic scattering or nuclear scattering. In magnetic scattering, the magnetic moment of the neutron interacts with the magnetic moment on the atom, originating from unpaired electrons. These are distributed over the atom - over dimensions comparable to the neutron wavelength - resulting in an angle-dependent magnetic form factor, which can be manipulated by reorienting the electron spins via an external magnetic field, and which can also be calculated from electron densities. In nuclear scattering, the neutrons interact directly with the nucleus of an atom via the strong nuclear interaction, and this scattering contribution cannot be predicted from theory. For a small number of strongly absorbing nuclei, such as ${ }^{113} \mathrm{Cd},{ }^{149} \mathrm{Sm}$, or ${ }^{157} \mathrm{Gd}$, there is also resonance scattering (as opposed to the two types of potential scattering mentioned above) when the neutron and the nucleus forms a compound nucleus; this phenomenon will not be discussed here.

The probability of a neutron to interact with a nucleus is quantified via the cross section $\sigma$, which is measured in barns (b). The cross section consists of a term representing the probability of scattering, $\sigma_{\text {scatt }}$ and a term representing the probability of absorption by the nucleus, $\sigma_{a b s}$, so that

$$
\sigma=\sigma_{s c a t t}+\sigma_{a b s}
$$

The small neutron cross section for most materials has both advantages and disadvantages; among the latter, the foremost is that weak interaction also means that neutrons are difficult to detect. This will be discussed later on, in section 8 . On a positive note, the weak interaction simplifies theoretical treatment of neutron scattering, which is often carried out in the so-called Born approximation, or the kinematic scattering approximation, in which it is assumed that the beam traverses the sample unaffected by it. Hence, refraction, multiple scattering and beam extinction by scattering of the primary beam are all neglected.

\subsection{Scattering lengths}

The strength in the interaction of a neutron with a nucleus is given by the scattering length $b$, which is a phenomenological quantity, and which must be determined empirically, since it cannot be calculated.

The scattering lengths for the elements, or for specific isotopes of these, do not vary in a systematic manner with nucleus composition; they have been plotted for some elements in Figure 6, where also the scattering lengths of individual isotopes are indicated for $\mathrm{H}$ and $\mathrm{Ni}$. For non-absorbing nuclei, the scattering length is independent of the neutron wavelength. The differences in scattering length between hydrogen $\left({ }^{1} \mathrm{H}\right)$ and deuterium $\left({ }^{2} \mathrm{H}=\mathrm{D}\right)$ is essential for many applications in soft matter research. Nickel has a very rich variety in scattering length between different isotopes, which is exploited for neutron mirrors and many other aspects of neutron research in the solid state. The seemingly arbitrary variation in scattering length between nearby elements and isotopes is an advantage over X-ray scattering, which can provide similar structural information, but with X-rays the scattering 


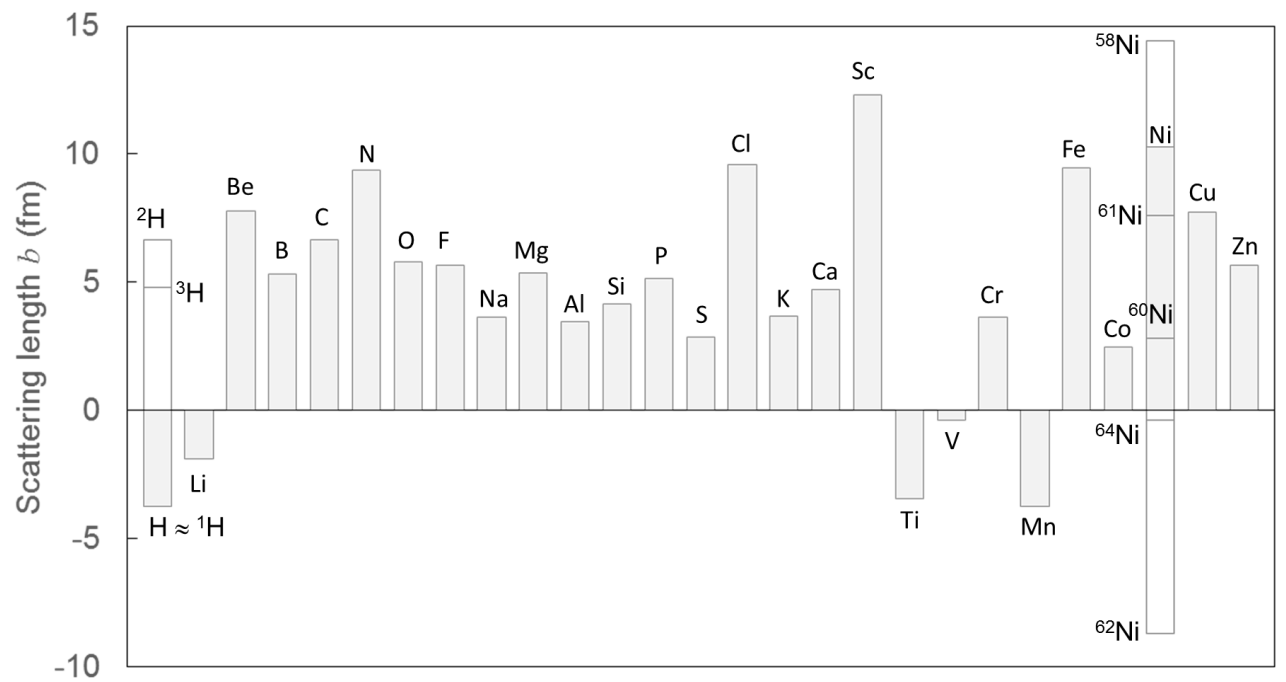

Figure 6. Neutron (bound, coherent) scattering lengths for some elements. For hydrogen and nickel, the scattering length of the elements in their natural compositions are indicated with the grey bars, but also the scattering length of specific isotopes are indicated with the transparent bars.

length is proportional to the density of electrons, meaning that nearby elements can be very difficult to distinguish due to the low contrast between them. The scattering length can be either positive or negative, and of significant importance for the use of neutrons to probe the structure of matter is the fact that hydrogen and deuterium have different signs of their scattering lengths, $b_{\mathrm{H}}=-3.74 \mathrm{fm}$ and $b_{\mathrm{D}}=6.67 \mathrm{fm}$, respectively, and that mixing these isotopes can be used to modulate the contrast in a system.

A free nucleus will not have the same scattering length as a bound nucleus, such as a nucleus restricted by a chemical bond or a lattice. The free $\left(b_{\text {free }}\right)$ scattering length is related to the bound scattering length $(b)$ via

$$
b_{\text {free }}=\frac{M}{M+m_{n}} b
$$

where $M$ is the nuclear mass and $m_{n}$ the neutron mass. Nuclei are rarely completely free, and in most cases bound scattering lengths are tabulated and used. From the relation above it is clear that free and bound scattering lengths are substantially different only for low mass elements, such as hydrogen, since the ratio $M /\left(M+m_{n}\right)$ approaches unity as $M$ becomes large.

The interaction of a wave with some medium - the potential that the wave will meet - depends on the local average scattering length of the atoms, but also on the number density of the scattering nuclei, and the product of the two is the scattering length density (SLD), and is given by

$$
\rho=\sum_{i} b_{i} n_{i}
$$

where $b_{i}$ is the scattering length of nuclear species $i$, and $n_{i}$ its number density. Scattering length densities of some materials are shown in Table 2. 
Table 2. Neutron scattering length densities (SLDs) for some materials. The right column includes solids often used as substrates in reflectometry.

\begin{tabular}{lclc} 
& $\mathrm{SLD}\left(10^{-6} \AA^{-2}\right)$ & & $\mathrm{SLD}\left(10^{-6} \AA^{-2}\right)$ \\
\hline $\mathrm{H}_{2} \mathrm{O}$ & -0.56 & $\mathrm{Si}$ & 2.07 \\
$\mathrm{D}_{2} \mathrm{O}$ & 6.38 & $\mathrm{SiO}_{2}$ & 3.39 \\
Octane & -0.53 & Quartz & 4.2 \\
$d$-octane & 6.43 & Sapphire & 5.4
\end{tabular}

The scattered intensity from inhomogeneities in the scattering length (densities), arising from, say molecules $\mathrm{X}$ in a solvent of $\mathrm{Y}$, is proportional to the square of the difference in SLD, sometimes called the contrast factor $\Delta \rho^{2}$ :

$$
\Delta \rho^{2}=\left(\rho_{X}-\rho_{Y}\right)^{2}
$$

\subsection{The refractive index}

The optical properties of visible light, and its interaction with matter, are usually described in terms of angle of incidence $\theta$ and refractive index $n$, but in neutron scattering it is customary to use scattering length density $\rho$ and momentum transfer $q$, since these are easily related to the reciprocal quantities that are natural for scattering studies. The mean scattering length density $\rho$ is related to the refractive index $n$ as:

$$
n=1-\frac{\lambda^{2}}{\pi} \rho+i \lambda C
$$

The constant $C$ in the imaginary term is a function of the absorption cross section of the material, but with the exception of a few strongly absorbing substances, such as cadmium, samarium, or gadolinium, the absorption is very small and this term is usually neglected. Magnetic materials have a contribution to the refractive index also from the neutron spin, which can be used in conjunction with polarised neutrons to study magnetism, though this is not considered here. The weak interaction of neutrons with matter means that the refractive index is usually very close to unity, with the quantity $(n-1)$ of the order of $10^{-6}$ for most materials. Note also that a positive scattering length implies that $n<1$.

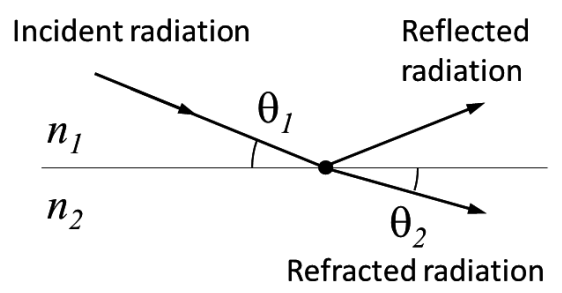

Figure 7. The geometry for the reflection and refraction of a beam incident on a surface at an angle $\theta_{1}$. The reflected beam leaves the surface at an angle $\theta_{1}$, while the latter is refracted at and angle $\theta_{2}$.

At an interface separating two media with refractive indices $n_{1}$ and $n_{2}$, the ratio of the refractive indices will determine the angle $\left(\theta_{2}\right)$ at which radiation incident at an angle $\theta_{1}$ will be refracted. With the conventions according to Figure 7, Snell's law gives:

$$
n_{1} \cos \theta_{1}=n_{2} \cos \theta_{2}
$$

If $n_{2}>n_{1}$, then $\theta_{2}>\theta_{1}$ and there exists a real angle of refraction, $\theta_{2}$, for all incidence angles $\theta_{1}$. However, since most elements have positive scattering lengths, then according to equation (3), the 


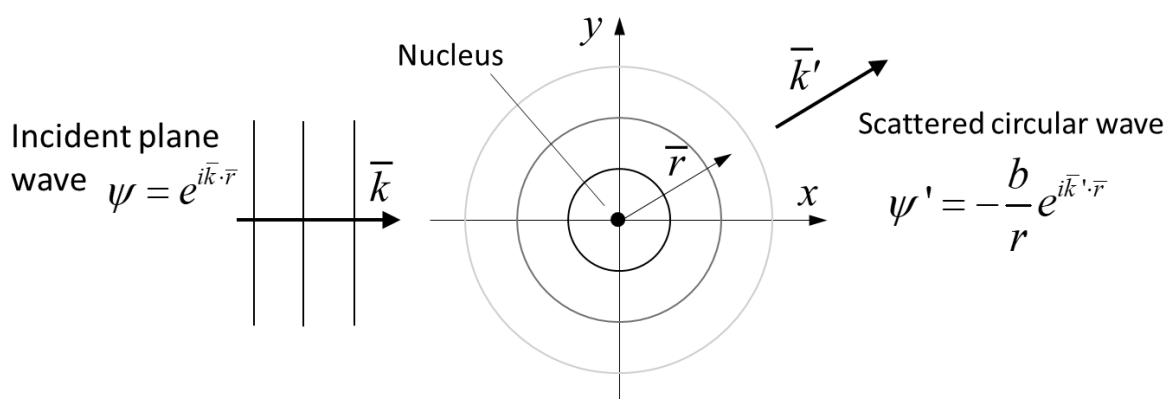

Figure 8. A wave $\psi$ with unit amplitude travelling in the positive $x$ direction with wavenumber $k=|\bar{k}|$ encounters a point scatterer (meaning that the size of the object is much less than the wavelength). The wave is scattered spherically, with an amplitude $b / r$, where $r$ is the distance from the scatterer (placed at $r=0$ ). The positive constant $b$ is the scattering length of the nucleus, and is a measure of the strength of the interaction. If the scattering is elastic, then $|\bar{k}|=\left|\bar{k}^{\prime}\right|$. (After Pynn [27].)

neutron refractive index is $<1$, and whenever material 1 is air or vacuum, $n_{2}<n_{1}$, and there is a real angle of refraction only for incidence angles larger than a critical angle $\theta_{c}$, at which the angle of refraction is 0 , and:

$$
\cos \theta_{c}=\cos \theta_{1}=\frac{n_{2}}{n_{1}}
$$

Where $\theta_{1}<\theta_{c}$, total external reflection occurs, and only an evanescent wave penetrates into material 2 . With the ratio $n_{2} / n_{1}$ deviating very little from unity, the angles at which total external reflection is observed are consequently very small, typically $<0.1^{\circ}$.

As long as inhomogeneities in the scattering length density are small, it is the average scattering length density in a medium that is important, and it is thus possible to mix, for example, hydrogenated and deuterated substances to vary the average scattering length density in a structure or a solvent to obtain a certain refractive index. Isotopic substitution is indeed a common method of contrast variation, i.e. changing the refractive index of a substance, and due to the large differences in scattering length between hydrogen and deuterium, this is especially so with hydrogenated compounds. The scattering length densities for $\mathrm{H}_{2} \mathrm{O}$ and $\mathrm{D}_{2} \mathrm{O}$ are such that most materials have SLDs that fall between these two forms of water, and this applies also to many other hydrogenated solvents and substances. In particular the $\mathrm{H}_{2} \mathrm{O} / \mathrm{D}_{2} \mathrm{O}$ ratio can be selected to make specific parts of a sample "invisible" to the neutrons. This contrast matching is widely used to highlight different parts of a system, and by conducting experiments on the same system but in different contrasts, also details of very complex structures can be revealed, as will be discussed in more detail in the subsequent chapters.

There are also certain "null matrix" alloys that result in zero coherent scattering lengths. Ti $\left(b_{c}=\right.$ $-3.44 \mathrm{fm})$ and $\mathrm{Zr}\left(b_{c}=+7.16 \mathrm{fm}\right)$, or $\mathrm{Mn}\left(b_{c}=-3.75 \mathrm{fm}\right)$ and $\mathrm{Cu}\left(b_{c}=+7.72 \mathrm{fm}\right)$ are use to form such alloys that are useful for engineering purposes.

\subsection{Point scatterers}

Consider the scattering from a nucleus, placed at $\bar{r}=0$, which we initially define as a spinless isotope with real scattering length $b$. Since the atomic nuclei are much smaller than the wavelengths used for neutron scattering, the nuclei act as point scatterers, resulting in isotropic scattering. For an incident 

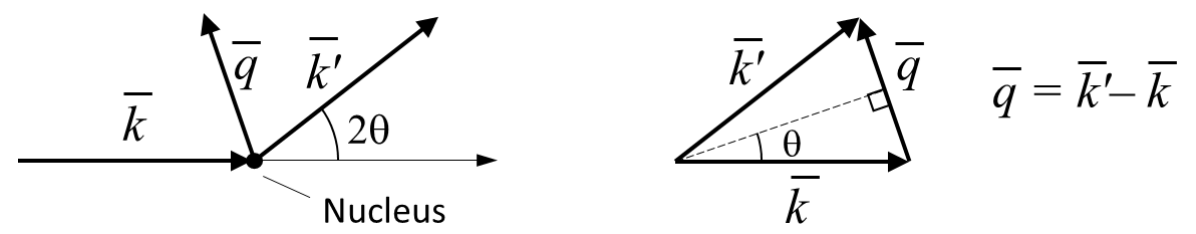

Figure 9. The relation between the momentum transfer $\bar{q}$ and the incident and scattered wavevectors, $\bar{k}$ and $\bar{k}^{\prime}$.

plane wave $\psi$, the scattered wave $\psi^{\prime}$ is then spherical (see Figure 8):

$$
\psi=e^{i \bar{k} \cdot \bar{r}} \text { and } \quad \psi^{\prime}=-\frac{b}{r} e^{i \bar{k}^{\prime} \cdot \bar{r}}
$$

The only (mathematically) possible potential giving rise to spherical (isotropic) scattering is a delta function, and we thus approximate the potential using the Fermi pseudopotential:

$$
V(\bar{r})=\frac{2 \pi \hbar^{2}}{m} b \delta(\bar{r})
$$

If we consider the potential from a group of $i$ particles, each with scattering length $b_{i}$ and at position $\bar{r}_{i}$, the resulting potential is

$$
V(\bar{r})=\sum_{i} \frac{2 \pi \hbar^{2}}{m} b_{i} \delta\left(\bar{r}_{i}\right)
$$

The scattering amplitude from this set of particles can be obtained via a Fourier transformation of this potential, yielding

$$
A(\bar{q})=\sum_{i} b_{i} e^{i \bar{q} \bar{r}_{i}}
$$

where $q$ is the scattering vector, or the momentum transfer, i.e. the difference between the scattered and the incident wavevectors, as shown in Figure 9. The magnitude of the scattering vector can also be expressed in angle of incidence $\theta$ and the wavelength $\lambda$ (keeping in mind that $k=|\bar{k}|=\left|\bar{k}^{\prime}\right|=2 \pi / \lambda$ ):

$$
\begin{gathered}
q=|\bar{q}|=\left|\bar{k}^{\prime}-\bar{k}\right|=\sqrt{\bar{k}^{\prime 2}+\bar{k}^{2}-2 \bar{k}^{\prime} \cdot \bar{k}}=\sqrt{k^{\prime 2}+k^{2}-2\left|\overline{k^{\prime}}\right||\bar{k}| \cos 2 \theta}= \\
=\sqrt{2 k^{2}(1-\cos 2 \theta)}=\sqrt{2 k^{2}\left(2 \sin ^{2} \theta\right)}=2 k \sin \theta=\frac{4 \pi \sin \theta}{\lambda}
\end{gathered}
$$

In an experiment, we measure the flux $J^{\prime}$ of neutrons scattered from the sample onto a detector subtending the solid angle $d \Omega=\sin \theta d \theta d \phi$ in the direction $(\theta, \phi)$, see Figure 10. Here, $2 \theta$ is the scattering (deflection) angle ${ }^{2}$, and $\phi$ is the azimuthal angle, i.e. the direction defined by the angle rotating around the incident wave direction. If the incident flux is $J$, then the ratio $J^{\prime} / J$ represents the fraction of neutrons scattered into the solid angle $d \Omega$, and it defines the differential scattering cross section:

$$
\frac{d \sigma}{d \Omega} \equiv \frac{J^{\prime}}{J}
$$

\footnotetext{
${ }^{2}$ This is the convention used in, for example, reflectometry, where $\theta$ is the incidence angle. In small-angle scattering, the scattering angle (the angle formed between $\bar{k}$ and $\bar{k}^{\prime}$ ) is $\theta$, and the equations involving the scattering angle adjusted accordingly.
} 


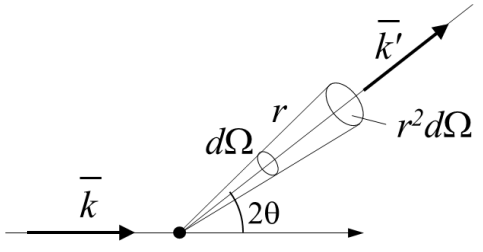

Figure 10. Geometry of a scattering experiment. The azimuthal angle $\phi$ rotates in the plane perpendicular to the incident wavevector $\bar{k}$.

If $J$ is a plane wave it represents the transmitted energy per unit area per second, while the spherical wave $J^{\prime}$ is the transmitted energy per solid angle per second. The ratio $J^{\prime} / J$ then has units of area per solid angle. Integrating $d \sigma / d \Omega$ over all solid angles yields the total scattering cross section:

$$
\sigma=\int \frac{d \sigma}{d \Omega} d \Omega
$$

Expressed in the waves $\psi$ and $\psi^{\prime}$, the incident flux is $v|\psi|^{2}$ and the total scattered flux into a sphere of radius $r$ is $4 \pi r^{2} v\left|\psi^{\prime}\right|^{2}$, where $v$ is the neutron speed, and with $\sigma$ defined as the scattered flux/incident flux, this gives

$$
\sigma=\frac{4 \pi r^{2} v\left|\psi^{\prime}\right|^{2}}{v \psi^{2}}=\frac{4 \pi r^{2} v\left|-\frac{b}{r} e^{i k^{\prime} r}\right|^{2}}{v\left|e^{i k x}\right|^{2}}=4 \pi b^{2}
$$

For elastic scattering where $|\bar{k}|=\left|\bar{k}^{\prime}\right|$ we have

$$
d \sigma(\bar{q})=\frac{J^{\prime} r^{2} d \Omega}{J}=|A(\bar{q})|^{2} d \Omega
$$

and within the Born approximation, this becomes

$$
\frac{d \sigma}{d \Omega}(\bar{q})=|A(\bar{q})|^{2}
$$

\subsection{Coherent and incoherent scattering}

If we insert (6) into equation (8), and take into account that the nuclei $b_{i}$ can be different isotopes, as well as have different nuclear spins, this results in a variation of scattering lengths, and to obtain the scattering amplitude from an array of $N$ particles we need to average over the distribution of scattering lengths:

$$
\frac{d \sigma}{d \Omega}(\bar{q})=|A(\bar{q})|^{2}=\left\langle\sum_{i}^{N} b_{i} e^{i \bar{q} \bar{r}_{i}} \sum_{j}^{N} b_{j} e^{-i \bar{q} \bar{r}_{i}}\right\rangle=\left\langle\sum_{i, j}^{N} b_{i} b_{j} e^{i \bar{q} \bar{r}_{i, j}}\right\rangle=\sum_{i, j}^{N}\left\langle b_{i} b_{j} e^{i \bar{q} \bar{r}_{i, j}}\right\rangle
$$

The scattering lengths $b_{i}$ and $b_{j}$ depend on the isotope and the spin state of each nucleus, and since they are independent of each other, the product of their expectation values is equivalent to the expectation value of their product. For the averages of the scattering lengths we obtain:

$$
\begin{gathered}
i \neq j: \quad\left\langle b_{i} b_{j}\right\rangle=\left\langle b_{i}\right\rangle\left\langle b_{j}\right\rangle=\left\langle b_{i}\right\rangle^{2}=\langle b\rangle^{2} \\
i=j: \quad\left\langle b_{i} b_{j}\right\rangle=\left\langle b_{i}^{2}\right\rangle=\left\langle b^{2}\right\rangle
\end{gathered}
$$

To rewrite the result for $i=j$ we use the relation

$$
\left\langle(b-\langle b\rangle)^{2}\right\rangle=\left\langle b^{2}-2 b\langle b\rangle+\langle b\rangle^{2}\right\rangle=\left\langle b^{2}\right\rangle-\langle b\rangle^{2} \Rightarrow\left\langle b^{2}\right\rangle=\langle b\rangle^{2}+\left\langle(b-\langle b\rangle)^{2}\right\rangle
$$


Inserting this into equation (9), we obtain (noting that $\bar{r}_{i, j}=0$ for $i=j$ ):

$$
\sum_{i, j}^{N}\left\langle b_{i} b_{j} e^{i \bar{q} \bar{r}_{i, j}}\right\rangle=\sum_{i \neq j}^{N}\langle b\rangle^{2}\left\langle e^{i \bar{q} \bar{r}_{i, j}}\right\rangle+\sum_{i=j}^{N}\langle b\rangle^{2}+\sum_{i=j}^{N}\left\langle(b-\langle b\rangle)^{2}\right\rangle
$$

Now, the first two terms on the right are in $\langle b\rangle^{2}$ and can be combined into a sum for all $\{i, j\}$, and after again applying relation (10) to the last term we obtain

$$
\frac{d \sigma}{d \Omega}(\bar{q})=\langle b\rangle^{2} \sum_{i, j=1}^{N}\left\langle e^{i \bar{q} \bar{r}_{i, j}}\right\rangle+N\left(\left\langle b^{2}\right\rangle-\langle b\rangle^{2}\right)
$$

From (12) we see that the scattering cross section has two contributions, the first term on the right still contains the phase factors $e^{i \bar{q} \bar{r}}$, and corresponds to scattering from nuclei with the same value of the average $\langle b\rangle$. This is a coherent superposition of pairs of scatterers, and takes into account all interference effects, and hence contains the structural information. This is the scattering we would obtain if all the scattering lengths were exactly $\langle b\rangle=b_{c}$, the coherent scattering length. The second term on the right does not contain phase information and hence does not result from interference, but from the random distribution of the actual scattering lengths in the system. This is the incoherent contribution, i.e. scattering from single atoms that superimpose in an incoherent manner. For a single isotope with zero nuclear spin, there is thus no incoherent contribution, but for other atoms, the total scattering cross section $\sigma_{\text {scatt }}$ has two contributions, so that $\sigma_{\text {scatt }}=\sigma_{c}+\sigma_{i}$, and can now write the coherent and incoherent contributions to the scattering cross section as

$$
\begin{gathered}
\sigma_{c}=4 \pi\langle b\rangle^{2}=4 \pi b_{c}^{2} \\
\sigma_{i}=4 \pi\left(\left\langle b^{2}\right\rangle-\langle b\rangle^{2}\right)=4 \pi b_{i}^{2}
\end{gathered}
$$

from which it is clear that the magnitude of $\sigma_{i}$ is determined by the mean square deviation of the scattering lengths. These relations hold under the assumption that there is no correlation between the scattering lengths and the positions of the nuclei, and are valid for spin and isotope incoherence. The situation where (more or less) random variations in scattering potential arise as a result of, for example, mixing of different components in a system, is referred to as diffuse scattering, even though this is also incoherent if the variations are genuinely random.

The coherent and incoherent contributions can be considered from a different perspective. The combination of a nucleus with non-zero spin $I$ and the neutron with spin $1 / 2$ can have two spin states, either parallel $(I+1 / 2)$ or antiparallel $(I-1 / 2)$, and these will result in different scattering lengths; $b_{+}$and $b_{-}$, respectively, and with different degeneracies. For $b_{+}$there are $2 I+2$ possible states, and for $b_{-}$there are $2 I$ states, and thus $4 I+2$ states in total. In calculating the total scattering length, the contributions from $b_{+}$and $b_{-}$must be weighted accordingly, with the weighting factors

$$
w_{+}=\frac{2 I+2}{4 I+2}=\frac{I+1}{2 I+1} \quad \text { and } \quad w_{+}=\frac{2 I}{4 I+2}=\frac{I}{2 I+1}
$$

Values of $b_{+}$and $b_{-}$are available for a limited number of isotopes, but for a particular isotope with mass number $A$, the means of $b$ and $b^{2}$ are obtained via

$$
\begin{gathered}
\langle b\rangle_{A}=w_{+} b_{+}+w_{-} b_{-}=\frac{(I+1) b_{+}+I b_{-}}{2 I+1} \\
\left\langle b^{2}\right\rangle_{A}=w_{+} b_{+}^{2}+w_{-} b_{-}^{2}=\frac{(I+1) b_{+}^{2}+I b_{-}^{2}}{2 I+1}
\end{gathered}
$$


If these means are again averaged over the isotopes for an element, with proper weight for their relative abundance, $\langle b\rangle$ and $\left\langle b^{2}\right\rangle$ can be calculated, and we have, as above, $b_{c}=\langle b\rangle$, and

$$
b_{i}=\sqrt{\left\langle b^{2}\right\rangle-\langle b\rangle^{2}}=\sqrt{w_{+} w_{-}}\left(b_{+}-b_{-}\right)
$$

If these relations are inverted, we can calculate $b_{+}$and $b_{-}$from $b_{c}$ and $b_{i}$ :

$$
b_{+}=b_{c}+\sqrt{\frac{I}{I+1}} b_{i} \text { and } b_{-}=b_{c}-\sqrt{\frac{I+1}{I}} b_{i}
$$

Calculating the coherent and incoherent contributions from assemblies of elements (molecules, solutions, etc.) involves a similar weighting procedure. Consider the compound $\mathrm{X}_{m} \mathrm{Y}_{n}$ with $m \mathrm{X}$ atoms and $n \mathrm{Y}$ atoms. For these, we use the weighting factors (number fractions) $w_{X}=m /(m+n)$ and $w_{Y}=n /(m+n)$, from which the coherent scattering cross section (per atom) is

$$
\sigma_{c}\left(\mathrm{X}_{m} \mathrm{Y}_{n}\right)=4 \pi\langle b\rangle^{2}=4 \pi\left(w_{X} b_{X}+w_{Y} b_{Y}\right)^{2}
$$

For example, for water, this yields $\sigma_{c}\left(\mathrm{H}_{2} \mathrm{O}\right)=4 \pi\left[w_{\mathrm{H}} b_{\mathrm{H}}+w_{\mathrm{O}} b_{\mathrm{O}}\right]^{2} \mathrm{fm}^{2}=4 \pi[(2 / 3)(-3.739)+$ $(1 / 3) 5.803]^{2}=0.039$ barn per atom, on average, or $3 \cdot 0.039=0.117$ barn per water molecule. A similar calculation for $\mathrm{D}_{2} \mathrm{O}$ gives 15.3 barn per molecule. Incoherent contributions are calculated similarly; the spin and isotope incoherence contributions for the compound $\mathrm{X}_{m} \mathrm{Y}_{n}$ are obtained from

$$
\begin{gathered}
\sigma_{i, s p i n}\left(\mathrm{X}_{m} \mathrm{Y}_{n}\right)=w_{X} \sigma_{i}(\mathrm{X})+w_{Y} \sigma_{i}(\mathrm{Y}) \\
\sigma_{i, \text { isotope }}\left(\mathrm{X}_{m} \mathrm{Y}_{n}\right)=4 \pi\left(\left\langle b^{2}\right\rangle-\langle b\rangle^{2}\right), \text { where }\langle b\rangle=w_{X} b_{X}+w_{Y} b_{Y} \text { and }\left\langle b^{2}\right\rangle=w_{X} b_{X}^{2}+w_{Y} b_{Y}^{2}
\end{gathered}
$$

After simplification, these two contributions yield the resulting incoherent cross section per atom:

$$
\sigma_{i}\left(\mathrm{X}_{m} \mathrm{Y}_{n}\right)=w_{X} \sigma_{i}(\mathrm{X})+w_{Y} \sigma_{i}(\mathrm{Y})+4 \pi w_{X} w_{Y}\left(b_{X}-b_{Y}\right)^{2}
$$

Again, for illustration, inserting the numbers for $\mathrm{H}_{2} \mathrm{O}$ and $\mathrm{D}_{2} \mathrm{O}$ gives 168.1 and 4.17 barn per molecule, respectively, showing the huge differences in the coherent and incoherent contributions for $\mathrm{H}_{2} \mathrm{O}$ and $\mathrm{D}_{2} \mathrm{O}$.

It is of interest that for example Vanadium has a very small coherent contribution to the scattering cross section, so that the entire contribution to $\sigma_{\text {scat }}$ is incoherent. This is not wavelength dependent, and Vanadium is thus used for calibration purposes, to determine the flux profile as a function of the wavelength.

For structural characterization, the incoherent scattering, which does not contain any structural information, adds a constant background, independent of $q$, and which is often determined emprically and then subtracted from the data. In studies of dynamics of atoms or molecules with quasielastic or inelastic scattering, the incoherent scattering is exploited. Since it depends on the correlation between the positions of one nucleus at different times (self-correlation), it provides information about dynamics or diffusion, and is particularly useful for $\mathrm{H}$ due to its high incoherent contribution.

\subsection{Absorption}

Neutron absorption cross sections are small for most nuclei - often much smaller than the scattering cross section - so that absorption can in most cases be ignored. The most significant absorbing isotopes are ${ }^{3} \mathrm{He},{ }^{6} \mathrm{Li},{ }^{10} \mathrm{~B},{ }^{113} \mathrm{Cd}$, and ${ }^{157} \mathrm{Gd}$. These few strongly absorbing isotopes are used in various forms in neutron detectors and for neutron shielding. 
For most isotopes the absorption is proportional to the wavelength, so that $\sigma_{a b s}(\lambda) \propto \lambda$, though this does not apply to some absorbing isotopes, such as ${ }^{113} \mathrm{Cd}$, and ${ }^{157} \mathrm{Gd}$, which are strong resonant absorbers in the thermal neutron energy range, with sharp absorption peaks of very high cross section. If these materials are needed, but not for their absorption properties so that absorption is to be avoided, there are often isotopes which have low absorption, for example ${ }^{11} \mathrm{~B}$ and ${ }^{160} \mathrm{Gd}$. Common neutron absorbing materials are made of $\mathrm{Cd}$ sheets, $\mathrm{B}_{4} \mathrm{C}$, or borated aluminium.

Using Beer's law for transmission, $T=I / I_{0}=\exp \left(-N l \sigma_{a b s}\right)$, where $N$ is the number density of atoms, we obtain the thickness $l$ of a Cd sheet (using $\sigma_{a b s}=2520$ b) that is needed to absorb $99.9 \%$ of the neutrons in a beam to ca $0.6 \mathrm{~mm}$.

In contrast to X-rays, metals tend to be rather transparent to neutrons, with low absorption cross sections, but also with low scattering cross sections; aluminum and zirconium are two good examples, which also makes them useful as sample containers or window materials. Note that even though absorption of neutrons is small for many materials, they can still be activated, why some care must be taken in the handling of these materials immediately after exposure to neutrons.

\section{Detection}

The weak interaction of neutrons with matter poses particular challenges for neutron detection. In a detector, we want as many neutrons as possible to be counted, unless it is a beam monitor, in which case we want a low, but controllable effect of the measurement. Detectors may be grouped according to the working principles, or depending on their function and purpose. Neutrons have to be detected indirectly, via generation of secondary charged particles, and this can be done in slightly different ways: i) Absorption with prompt emission. Most detectors for scattering purposes fall into this category, and it includes prompt emission of detectable radiation from nuclides such as ${ }^{3} \mathrm{He},{ }^{6} \mathrm{Li}$, ${ }^{10} \mathrm{~B}$ and ${ }^{235} \mathrm{U}$. ii) Elastic scattering (proton recoil), where collisions cause ionization, which is easy to detect, or iii) via activation, that is, reaction with absorbing materials, which emit radiation at a later time. This is clearly not suitable for detectors in scattering experiments.

\subsection{Gas-filled detectors}

Gas-filled detectors are used for detection of thermal neutrons via various nuclear reactions (or for detecting fast neutrons via recoil interaction). These have a gas enclosed in a thin-walled metal tube cathode (typically made from aluminium), and are filled with isotopes such as ${ }^{3} \mathrm{He}$, or ${ }^{10} \mathrm{~B}$ in the form of $\mathrm{BF}_{3}$, and have an anode wire in the center of the tube, and with a high voltage applied between the anode and the cathode. An incident neutron causes a nuclear reaction, and the high kinetic energy of the reaction products ionizes gas atoms, which in turn ionize other gas atoms in an avalanche-like multiplication process. The induced charges result in a measureable electrical pulse. An important parameter for detectors is their gamma-ray sensitivity, and their capacity to distinguish gamma rays from neutrons, since gamma-ray generation can be considerable in the neutron beam.

In a ${ }^{3} \mathrm{He}$ detector, an incident neutron produces ${ }^{3} \mathrm{H}$ and ${ }^{1} \mathrm{H}$; the proton ionizes the surrounding gas atoms to create the charges that are detected by the counter.

Boron-filled detectors, in the form of gaseous $\mathrm{BF}_{3}$ are also used for detection of thermal neutrons, but are less sensitive than ${ }^{3} \mathrm{He}$ counters. However, the energy of the neutron reaction with $\mathrm{BF}_{3}$ is higher than for ${ }^{3} \mathrm{He}$, allowing better discrimination against gamma radiation.

These counters are used in a voltage range where the current pulse is proportional to the energy deposited in the gas - not the incident neutron energy, but to the number of captured neutrons - and hence they are called proportional counters. It is also common to add additional gases to improve 
performance; a heavy gas (such as Ar) reduces the range of the fission products, and ensures that more of their kinetic energy is deposited in the gas (and not in the container walls). This will also improve the charge collection time but also increase sensitivity to gamma rays. Polyatomic gases, such as methane or carbon dioxide in ${ }^{3} \mathrm{He}$ counters, act as quenchers in that they dampen the avalanche process by taking up kinetic energy in rotational modes, and improve the resolution.

Counters based on ${ }^{3} \mathrm{He}$ have been widespread for long, but supply of ${ }^{3} \mathrm{He}$ has been limited in recent years; ${ }^{3} \mathrm{He}$ is obtained as a decay product of tritium, which in itself is a byproduct of nuclear reactors or from weapons programs. Increased demand of radiation detectors for security, and decreasing supply, lead to the "Helium-3 crisis" in 2009, with soaring prices and reduced availability. Since then, replacement technologies have been a very hot topic in detector development.

Boron-lined gas-filled proportional counters react similarly to $\mathrm{BF}_{3}$ gas-filled proportional detectors. Detection is by means of a ${ }^{10} \mathrm{~B}$ coating rather than boron or ${ }^{3} \mathrm{He}$ gas, enabling a higher neutron sensitivity.

Recently, boron carbide $\left(\mathrm{B}_{4} \mathrm{C}\right)$ has been proposed as an alternative liner material for gas-filled counters, where aluminium blades are coated with a thin film containing high amounts of ${ }^{10} \mathrm{~B}$.[28]

A fission chamber is a type of gas-filled detector where some fissionable material (typically ${ }^{235} \mathrm{U}$ ) is coated as a thin film inside the chamber, and this layer is exposed to some detector gas (alternatively, it is filled with $\mathrm{UF}_{6}$ gas). After a neutron has induced a fission event, fission fragments travel in nearly opposite directions, and the ionization from the fragment that enters the gas is sensed by the detector. The detection efficiency is rather low, but these detectors are thus useful as beam monitors, where the intensity, or the white beam spectrum, of the beam incident on a sample is measured, and it is desired that the attenuation should be low. Monitors are also made from low-pressure ${ }^{3} \mathrm{He}$ cells.

Proportional counters are made position-sensitive by using a resistive center wire, instead of a good conductor. In this case, the charge generated by an incident neutron can be measured at both ends of the wire, and the charge mesured at either end is proportional to the distance from the charging event, and hence the location of the incident neutron can be determined. By stacking a large number of such position-sensitive counters, a two-dimensional position-sensitive neutron detector is obtained.

\subsection{Scintillation detectors}

Scintillator-based detectors use absorbing isotopes in a salt mixed with some scintillator material, for example ${ }^{6} \mathrm{LiF}$ or ${ }^{10} \mathrm{~B}_{2} \mathrm{O}_{3}$ used with the scintillating $\mathrm{ZnS}$, or $\mathrm{LiCaAlF}_{6}$ mixed with a scintillating dopant like Cesium or Europium. An incident neutron causing a reaction in the absorbing isotope will emit radiation which is converted to light by the scintillator material, and a photodetector/photomultiplier records the generated light. It is essential that the scintillator material is transparent to the emitted radiation; if emitted photons are re-absorbed, this will reduce the efficiency. Scintillators are generally both faster and have better spatial resolution than gas detectors, but have much higher sensitivity to gamma rays. The more compact design, however, gives them several advantages over gas detectors in arrays or two-dimensional sensors. An Anger camera is a two-dimensional scintillation detector with one single slab of scintillator material, placed in front of an array of photomultipliers. An incident neutron will generate light on several detectors, and by analysing the photodetector signals, the location of this event can be determined to within a few millimeters.

\section{Acknowledgements}

I thank the organizers of the 2016 French-Swedish Winter School on Neutrons and Soft Matter and J. Oberdisse for critical reading of this chapter, and B. Nagy for discussions and comments. 


\section{References}

[1] A. Pais, Inward bound: Of Matter and Forces in the Physical World (Clarendon, Oxford, 1988)

[2] J.J. Thomson, Philosophical Magazine Series 6 7, 237 (1904)

[3] H. Geiger, E. Marsden, Proceedings of the Royal Society of London. Series A 82, 495 (1909)

[4] E. Rutherford, Philosophical Magazine Series 6 21, 669 (1911)

[5] E. Rutherford, Proceedings of the Royal Society of London. Series A 97, 374 (1920)

[6] H. Becker, W. Bothe, Zeitschrift für Physik 76, 421 (1932)

[7] I. Joliot-Curie, F. Joliot, Comptes Rendus 194, 876 (1932)

[8] J. Chadwick, Nature 129, 312 (1932)

[9] J. Chadwick, Proceedings of the Royal Society of London. Series A 136, 692 (1932)

[10] H. von Halban, P. Preiswerk, Comptes Rendus de l'Académie des Sciences (Paris) 203, 73 (1936)

[11] D.P. Mitchell, P.N. Powers, Physical Review 50, 486 (1936)

[12] F. Bloch, Physical Review 50, 259 (1936)

[13] E.O. Wollan, C.G. Shull, Physical Review 73, 830 (1948)

[14] C.G. Shull, E.O. Wollan, Physical Review 81, 527 (1951)

[15] B.N. Brockhouse, A.T. Stewart, Physical Review 100, 756 (1955)

[16] D.M. Engelman, P.B. Moore, Proceedings of the National Academy of Sciences 69, 1997 (1972)

[17] R.G. Kirste, W.A. Kruse, J. Schelten, Die Makromolekulare Chemie 162, 299 (1972)

[18] J.P. Cotton, D. Decker, H. Benoit, B. Farnoux, J. Higgins, G. Jannink, R. Ober, C. Picot, J. des Cloizeaux, Macromolecules 7, 863 (1974)

[19] J.B. Hayter, R.R. Highfield, B.J. Pullman, R.K. Thomas, A.I. McMullen, J. Penfold, Journal of the Chemical Society, Faraday Transactions 1: Physical Chemistry in Condensed Phases 77, 1437 (1981)

[20] G.E. Bacon, Fifty years of neutron diffraction : the advent of neutron scattering (Hilger, Bristol, 1987)

[21] J. Reijonen, Proceedings of the 2005 Particle Accelerator Conference pp. 49-53 (2005)

[22] F. Mezei, Physica B: Condensed Matter 234, 1227 (1997)

[23] B. Willis, C. Carlile, Experimental neutron scattering (Oxford University Press, Oxford, 2009)

[24] H. Maier-Leibnitz, T. Springer, Reactor Science and Technology 17, 217 (1963)

[25] F. Mezei, Communications on Physics 1, 81 (1976)

[26] J. Stahn, U. Filges, T. Panzner, The European Physical Journal - Applied Physics 58, 11001 (2012)

[27] R. Pynn, Neutron Scattering - A Non-destructive Microscope for Seeing Inside Matter (Springer US, Boston, MA, 2009), pp. 15-36

[28] C. Höglund, J. Birch, K. Andersen, T. Bigault, J.C. Buffet, J. Correa, P.v. Esch, B. Guerard, R. Hall-Wilton, J. Jensen et al., Journal of Applied Physics 111, 104908 (2012) 